\title{
The Development and Application of Intelligent Testing System for Relay
}

\author{
Protection Based on IEC61850 \\ Zhengming Chen ${ }^{1, a}$, Bing Cui ${ }^{2, b}$, Zhenjun Zhang ${ }^{3, c}$ \\ ${ }^{1}$ State Grid Jiangxi Electric Power Research Institute, Nanchang ,China \\ ${ }^{2}$ State Grid Jiangxi Electric Power Research Institute, Nanchang ,China \\ ${ }^{3}$ State Grid Electric Power Research Institute, Nanjing , China \\ achenzhengming163@163.com, ${ }^{b}$ wshrcb@163.com, ${ }^{c}$ zhangzhenjun@sgepri.sgcc.com.cn
}

Key words: Manufacturing Message Specification; Report-Control-Block; Setting-Group-Control-Block; ACSI ; intelligent testing

Abstract: We have developed the IED device's Intelligent Testing System for the lack of existing field-testing techniques. The system can automatic access to the tested IED's configuration parameters by simulating the MMS service. It can use the parameters to set the test parameters of the process layer and tests the IED. The system can also intelligently analyze behavior and results of IED using the MMS network packets. It has achieved the intelligent testing for intelligent substation's relay protection.

\section{Introduction}

IEC61850 ${ }^{[1]}$ transfers complex scene integration process into offline configuration procedure, and all configuration papers in intelligent transformer substation describe all information there, which includes transformer substation device topological structure, all intelligent device model and function and communication configuration among intelligent device. In the process of relay protection debugging in intelligent transformer substation, we spend most time on creation and modification of intelligent transformer substation configuration papers. Once modify configuration paper, we must test again. In process of testing, we need to protect its function.

IEC61850 uses layering, object-oriented modeling and other new techniques, which bottom is directly mapped to manufactured message standard (MMS ${ }^{[2]}$, supporting automatic testing. Although manufacturer has testing tools for testing accuracy of their own device configuration, these tools are for manufacturer's equipment, exclusively. So it needs to design and develop new testing system, which uses IEC61850 MMS communication property to develop intelligent software. This product can automatically get configuration parameter of IED device, so set testing parameter automatically and test IED device intelligently with university. The whole testing process is controlled by testing software without manual intervention, reducing testing work amount greatly and ensure testing accuracy. 


\section{Testing System Framework and Principle}

\section{System Framework}

The basic framework of developed relay protection intelligent testing system based on IEC6180 agreement is shown in fig.1.

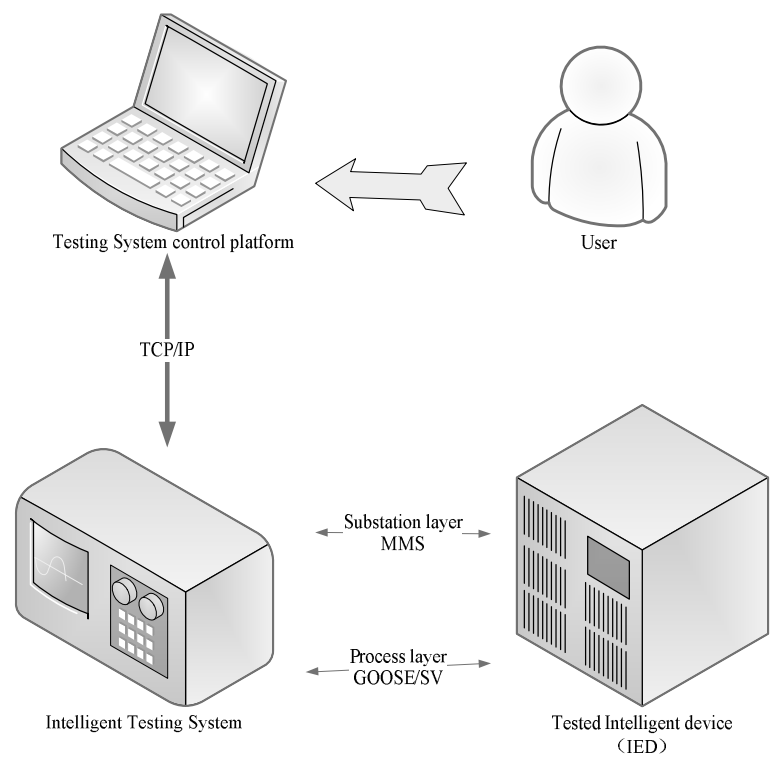

Fig. 1 Principle block diagram of intelligent testing system

Intelligent testing device uses MMS agreement to communicate with tested device, then acquiring tested device's setting parameter and communication parameter; later, it can set testing scheme and process layer data parameter, output SV/GOOSE and receive GOOSE information for carrying out testing on IED; after testing, we can get result from testing device with analysis result automatically. The whole test forms an automatically closed-loop system, getting parameter, setting scheme and analyzing result are all intelligent realization.

Intelligent testing software is developed based on MMS-EASE Lite ${ }^{[3]}$,it simulates that the client communicates with the smart IED.MMS-EASE Lite is from optimization and reduction of MMS-EASE software by SISCO company,it adds supporting for IEC61850 features. We use MMS-EASE Lite realizing IEC61850's mapping work to MMS in intelligent testing system.

Specific operating principle flow is shown in fig.2, which describes the whole testing flow.

\section{Testing Principle}

Each IED contains one or more LD(Logic Device). Each LD contains a LN0 and several LN(Logic Node). LN is the smallest functional unit, such as PTOC type's LN expresses overcurrent protection with time including the time and overcurrent protection setting and start status,and MMXU expresses an analog quantity of three-phase system including current and voltage and power etc, and CSWI expresses switch objects including the state and control point of switch etc. It is known by LN included in LD that LD can support function. LN's subordinate data is called DO(Data Objects). Meaning of each DO is defined uniformly in IEC 61850 standard,it also includes subordinate DO and DA(Data Attributes), and therefore DO can be seen as a structured data. DA is the final stage data, it also includes DA. DA information is divided into several types,it is marked by DA's function constraint attributes (FC). Common FC has the following categories:ST (status informating), $\mathrm{CO}$ (control),SG(settinggroup),SE(setting group editable), BR(buffered report control block), RP(unbuffered report control block), in which status 
class data includes strap status, binary input status, warning message, event information etc;

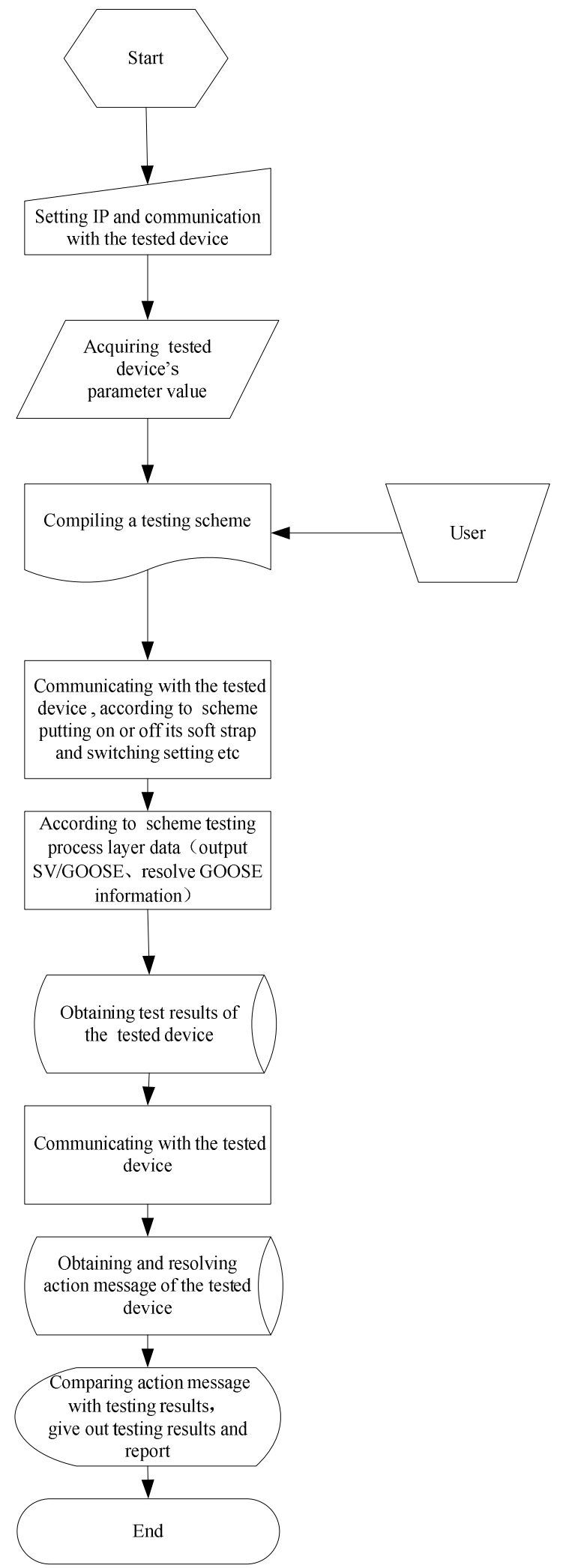

Fig. 2 Principle flowchart of intelligent testing system

Measuring class data includes the measure of measurement and control device and the measure of protection device; Control class data includes the control of reverting,strap and switch etc.Setting region includes each functional unit specific parameters,such as the impedance setting of distance protection etc.Such we call the service of setting, controlling, reporting, replacing by MMS, status 
quantity(functional constraint is ST), measuring quantity(MX) control quantity(CO) and setting(SG/SE).This is the current status parameters, Testing System configures testing scheme according to testing requirements.Such as after getting soft strap status Testing System modifies soft strap status and tests only single function of protection, thus unaffected by other functions; after getting setting of protection Testing System Intelligently sets testing parameters, thus reduce human molestation creating affection.

\section{Testing System Hardware Composition}

Testing system hardware consists of one testing supervisor and embedded board card. The testing supervisor uses ordinary IPC, which mainly completes testing system establishment, compiling testing scheme, testing task downloading and supervising testing flow. Look up and save testing result; embedded board card takes charge of real time data calculating and real time communication, involve process level data input and output, calculating model analytic calculation. Hardware framework is shown in fig.3.

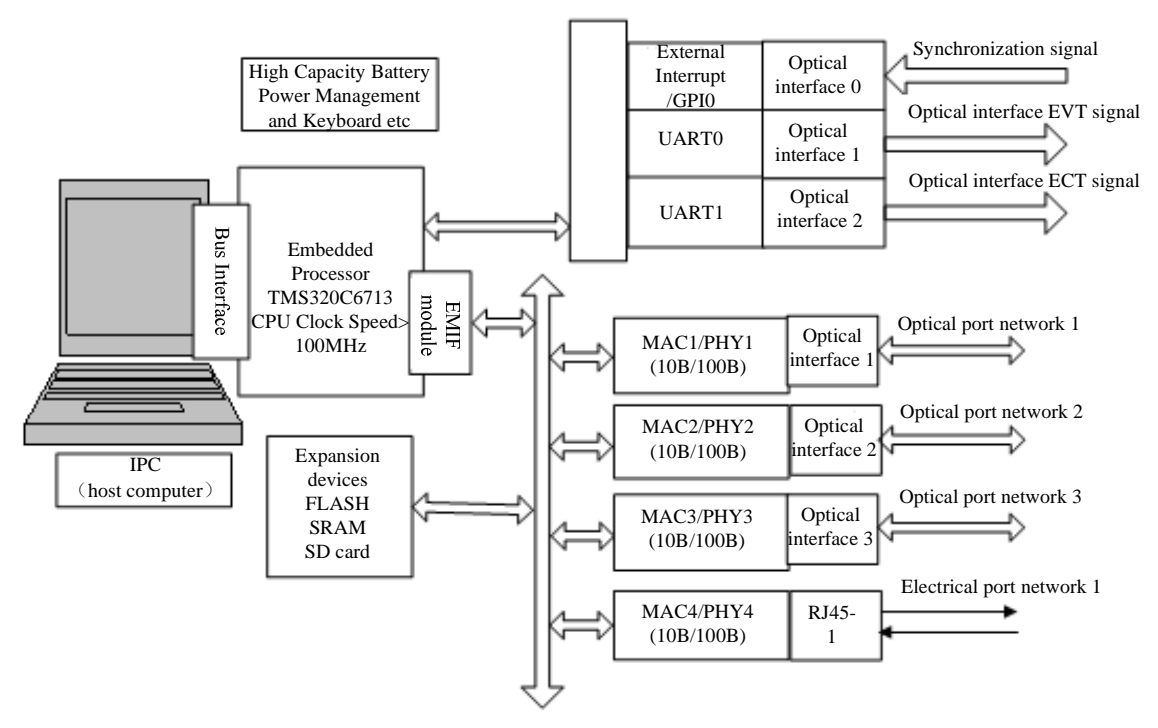

Fig. 3 Block diagram of intelligent testing system hardware

\section{Testing Scheme Intelligent Configuration}

In testing system, the information from tested device must be combined with tested device, so we can conduct intelligent test. Currently, tested device parameter in common testing instruments needs to be set by manual work, and testing content and method is determined by tester's experience. We set standard testing element library in intelligent testing procedure, such as impedance and low- frequency, which are corresponding with normal protection functional unit. As a result, tested device configuration information we have obtained can be used to form testing unit and parameter automatically, and then forming intelligent testing scheme. In addition, testing system also offers test case library to extend testing function. Testing system offers basic testing 
unit, such as frequency, amplitude and phase change, and tester forms a new testing scheme by set parameter which is added into library as a case. So each or a set of protection can be related to testing case. For next test, testing case and specific setting parameter are intelligently combined for automatically testing. With more and more testing cases, intelligent function of testing system becomes increasingly strong.

There is an example that intelligent testing system is used to test X7111 protection of Nanjing Xinning Photoelectricity Company, accounting for intelligent configuration process of testing scheme. Set IP address and build MMS communication to acquire protected setting of protected device. We may intelligently acquire protective functional unit by setting read interface, including phase-to-phase fault protection, ground fault protecting, zero-sequence protection, low- frequency and low-voltage protection, and the corresponding testing unit is distance protection setting, ground distance setting, zero-sequence protection and low-frequency load shedding and low voltage load shedding, shown in fig.4. Key words that Testing system intelligently acquires setting parameter, such as "distance", "zero-sequence", "low- frequency" and "low-voltage", form a test task group and a test configuration scheme, and test system automatically conducts X7111 protection setting and function test as sequence.

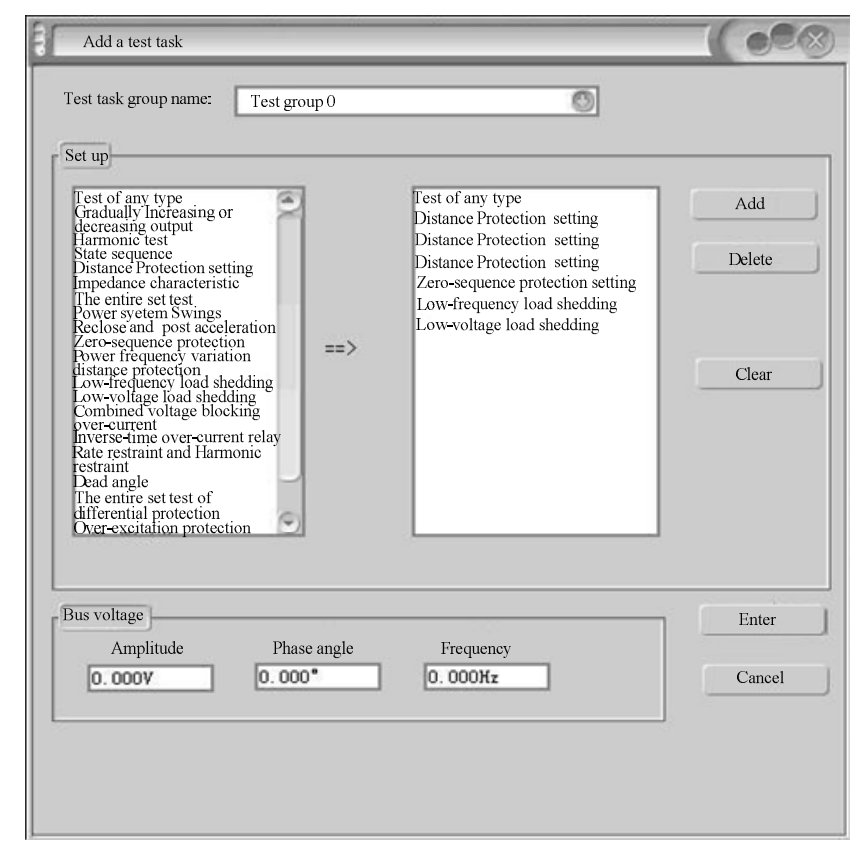

Fig. 4 Configuration figure of testing scheme

\section{Setting Group Automatically Control}

One of the key techniques in intelligent test is controlling for setting of tested IED device.

In logic equipment, there is a setting group control block SGCB, which includes NumOfSG, ActSG, EditSG and CnfSG. Thereinto, NumOfSG is the number of designated setting group, ActSG is active region (current setting group), EditSG is editing setting group, CnfSG is confirmed editing setting group.

In testing system software, there is a module for reading and writing setting, which function is to read tested device setting and modify it as need. This module uses SGCB ( setting group control 
block) interrelated service in tested device ACSI service to inquire and modify tested device setting. Reading setting aiming at tested device some LD, after user designates LD, setting reading module may acquire SGCB Reference under this LD, at this moment, it can acquire all setting under this LD and Reference related to each setting. Reading setting module flow chart is shown in fig.5, which includes two parts:

(1)setting reading module acquires all setting definition in tested device, showing setting definition;

(2)after showing setting, modify, submit and activate setting.

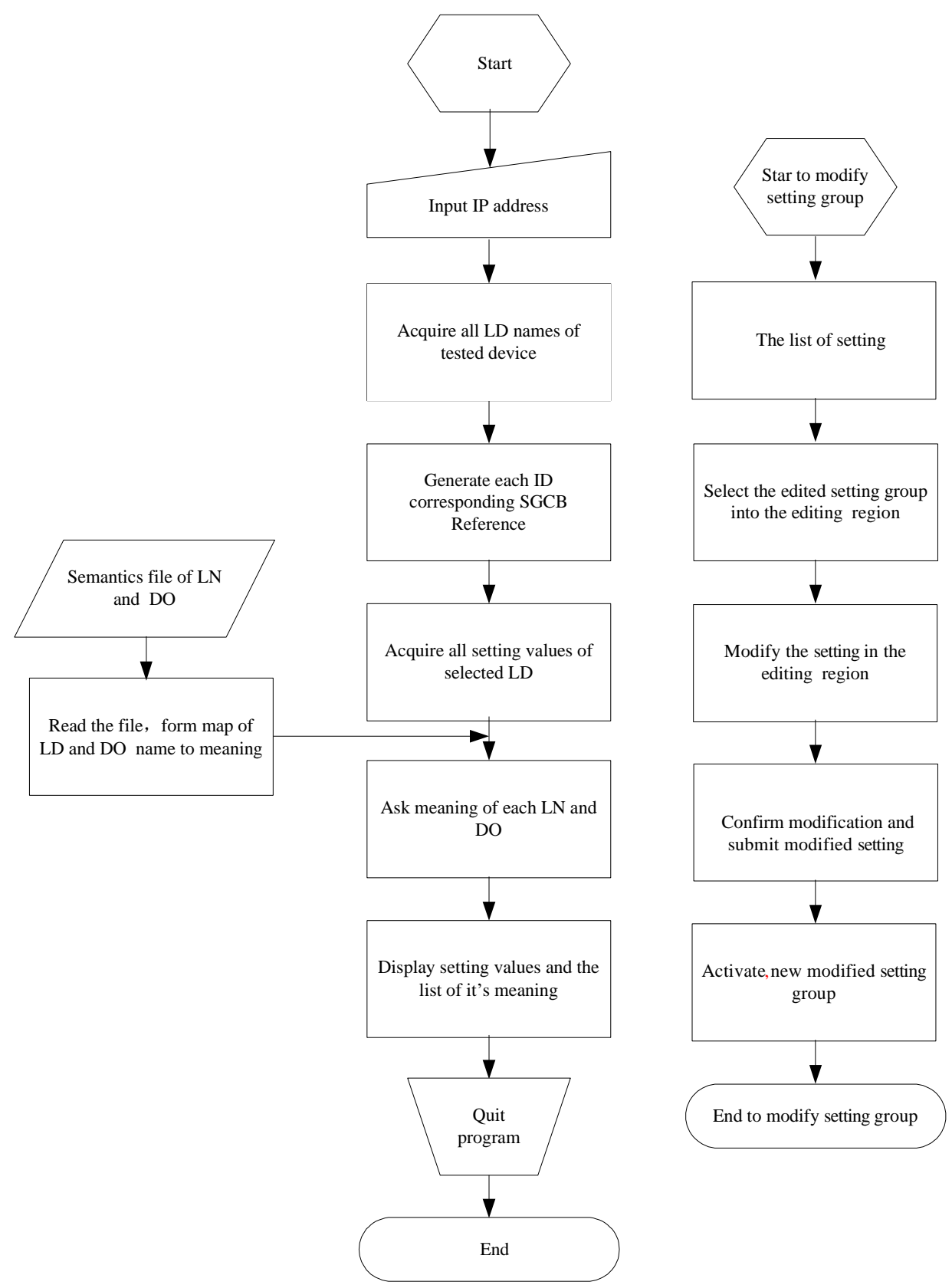

Fig. 5 Flowchart of module for reading and writing setting

\section{Intelligent Judgment for Testing Result}

After test, testing system gets two report results, one is testing result from testing system slave 
machine, the other is action report from tested device through MMS, shown in fig.6:

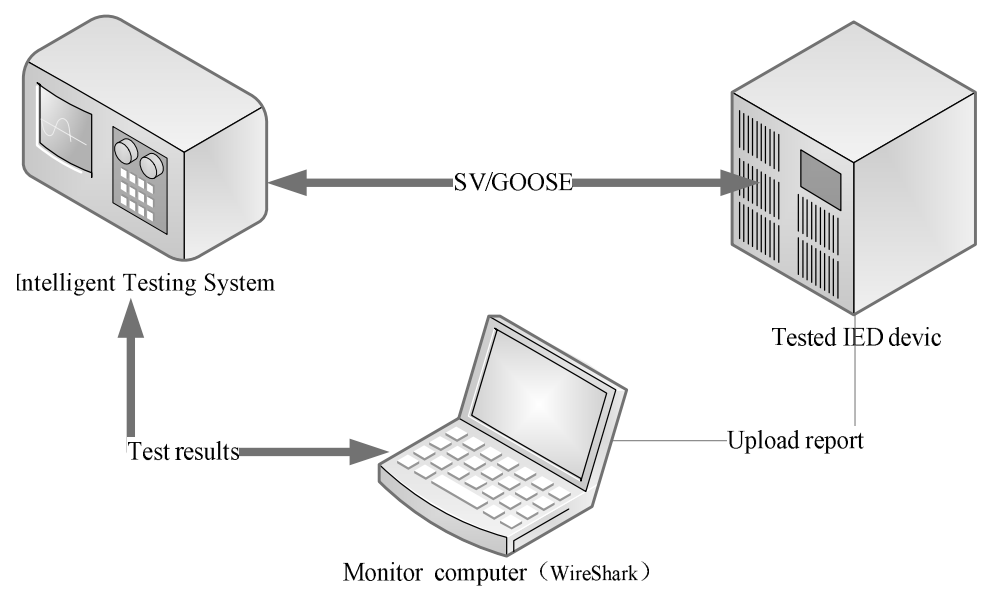

Fig. 6 Data of testing result

Testing system intelligently judges the differences and similarities between the two results, giving the specific conclusion whether the device is qualification and printing report. For achieving these functions, first, we can correctly unscramble the report from tested device, and we also use dictionary file pattern. Second, we compare with actual testing result from testing slave machine, or can related with it, simply, A phase action time must compare with A phase. But in fact, the format of every protection results are inconformity, it needs to select and judge intelligently. We formed different testing mother set according to different protection function. At last, the compared result is formed into word document for the convenience to modify and print by tester. Testing system may modify the format of word document according to tester's requirement, who only needs to offer its document template file.

\section{Conclusions}

(1) The testing system provides the application of process layer SV/GOOSE signal and station layer MMS for intelligent test of IED device.

(2) it can intelligently configure testing scheme, automatically set up test parameter and intelligently appraise test result,which builds a complete closed-loop intelligent testing system.

(3)it greatly reduces manual operation on testing scene and testing error probability, increases testing efficiency.

\section{References}

[1]ZHU Bingquan, WANG Song, LI Hui, LIU Jun , HUANG Xiaoming and JIANG Jianning: In Chinese ,Automation of Electric Power Systems, 2009, (8),p.104-107.

[2] WANG Dewen,ZHU Yongli,ZHAI Xueming,DI Jian: In Chinese,Automation of Electric Power Systems, 2008,32(22), p.56-60.

[3] WANG Shu-jing, YE Shen-mi, ZHANG Le: In Chinese,Instrumentation Technology,2009,(6), p.50-53. 